\title{
Editor's Note
}

\section{A SPECIAL ISSUE EDITED BY DR. LOUIS J. IGNARRO, A NOBEL PRIZE LAUREATE}

The distinguished Associate Editors and Section Editors of APR have worked with experienced internal as well as external referees to select and publish excellent articles in every issue of APR. APR publication is placing an emphasis on articles focusing on drug design, discovery and development.

In this August issue, we are pleased to publish the special review articles that focus on the theme of the chemistry and biology of nitric oxide (NO). As an introduction to this issue, Dr. Louis J. Ignarro (Nobel Prize Laureate, University of California Los Angeles and Konkuk University) has made valuable comments on the recent advances on the chemistry and biology of NO. Dr. Ignarro has also summarized the importance and significance of the presented review articles. We are confident that these reviews will be informative to APR readers particularly because $\mathrm{NO}$ and the associated signaling pathways play crucial roles in a number of biological events such as oxidative and nitrosative stress, cytoprotection, signal transduction, and neurotransmission. Since an excess amount of NO generated from inflammatory cells may increase oxidative and nitrosative stresses, thereby facilitating the pathologic process of a variety of diseases, the chemistry and biology of NO are closely linked with the discovery of potential drug targets.

As you know, APR aims to enhance scientific understanding in the field of pharmaceutical research, especially for drug discovery, development and elucidation of the mechanism of drug action. Thus, Prof. Young Choong Kim, the President of the Pharmaceutical Society of Korea reinforces the editorial members and advisory boards at the beginning of her presidency for global scientific expansion of APR. The current editorial members have made every effort to improve the journal's quality with the ultimate aim of advancing the applied sciences and new drug development. As a result of one of our recent efforts, the submission of APR manuscript and the peer review process are now operated only by the Springer on-line system, which surely facilitates the rapid publication of accepted papers and their exposure to scientific societies more efficiently. We hope that our endeavors help toward improving the quality of this journal among the SCI Expanded journals. To accomplish the promotion of APR to the SCI category, we need your continued academic contributions and support.

Sang Geon Kim, Ph.D.

Editor-in-Chief

Mi-Ock Lee, Ph.D.

Editor

Archives of Pharmacal Research

The Pharmaceutical Society of Korea

(THIS ISSUE IS FINANCIALLY SPONSORED BY DONG WHA PHARM. CO., LTD) 